\title{
Predominance of Gram-negative infections a cause of neonatal sepsis among low birth weight preterm infants
}

\author{
https://doi.org/10.1515/labmed-2020-0022 \\ Received February 28, 2020; accepted December 11, 2020; \\ published online January 21, 2021
}

\section{Abstract}

Objectives: Neonatal sepsis continues to be a leading and most important cause of mortality among low birth weight (LBW) preterm infants.

Methods: The study group included 226 LBW preterm infants with sepsis. A total of 100 normal birth weight infants with sepsis served as control. Blood and cerebrospinal fluid (CSF) samples collected for microbiological culture and processed according to standard protocol. Antibiotic resistance patterns were performed following standard guidelines.

Results: Out of 226 LBW preterm infants 106 (46.9\%) showed positive blood culture, of which 52 (49\%) Escherichia coli, 28 (26.4\%) Klebsiella pneumoniae, 12 (11.32\%) Enterobacter spp., 4 (3.7\%) Pseudomonas aeruginosa, 2 (1.88\%) Acinetobacter spp., 5 (4.7\%) methicillin-resistant Staphylococcus aureus (MRSA), 3 (2.8\%) Staphylococcus epidermidis were isolated. Out of 100 control group infants, $46(46 \%)$ showed positive blood cultures, of which 18 (39.1\%) E. coli, 12 (26.08\%) Enterobacter spp., 8 (17.39\%) K. pneumoniae, 1 (2.17\%) P. aeruginosa, 2 (4.34\%) MRSA, 3 (6.52\%) S. epidermidis and 2 (4.34\%) Candida spp. were isolated. Cephalosporin and Penicillin group showed highest resistance with 58 and 52\%, respectively. Aminoglycosides showed 22\% resistance. The control group showed highest resistance of $62 \%$ to the Penicillin group, $54 \%$ cephalosporin and 18\% for aminoglycosides.

Conclusions: Our study highlights on the surveillance of resistant pathogens causing sepsis in LBW preterm infants

*Corresponding author: Liyi Hu, Department of Clinical Laboratory, The First People's Hospital of Chongqing, Liang Jiang New Area, No. 199 Renxing Road, Renhe Street, Chongqing 401121, P.R. China, Phone/Fax: +86 13277652399, E-mail: ljxqyyjyk@163.com Xinlu Bai, Taimei Duan, Yuling Yi and Hong Peng, Department of Clinical Laboratory, The First People's Hospital of Chongqing, Liang Jiang New Area, Chongqing, P.R. China

Qiang Wei, Department of Clinical Laboratory, Children's Hospital of Chongqing Medical University, Chongqing, P.R. China emphasizing antimicrobial stewardship to control the mortality rate.

Keywords: antibiotic resistance; infection; neonatal sepsis; pathogens; preterm newborns.

\section{Introduction}

Neonatal sepsis is a leading cause of morbidity and mortality among low birth weight preterm infants (LBWI) [1-3]. The causative organisms influence the risk of complication. LBW preterm infants are more prone for infections mainly because of their underdeveloped immune systems. Prolonged hospital stay and central venous catheterization are also other main cause of Neonatal sepsis in LBW preterm infants $[4,5]$. Among developed countries an incidence rate of $10-25 \%$ of LBW preterm infants developing sepsis had been reported [6]. In a study $35 \%$ of mortality was reported among new born with sepsis in low birth weight infants compared to $11 \%$ mortality in uninfected low birth weight infants [7].

Neonatal sepsis with known risk of mortality the survivors of neonatal sepsis had been reported to develop more severe morbidity like pulmonary hypertension, respiratory shock and failure [8]. A change in the pathogen distribution has been reported by Neonatal Research Network among the low birth weight infants born before 1993 and after 2000. Gram-positive microbes were found to be common pathogens during early periods but later Gramnegative microbes predominated.

Among neonates systemic infections caused by bacteria occurs due to predisposing factors such as prematurity, premature membrane rupture, poor maternal nutrition, low birth weight, maternal conditions, duration of life support procedures required for postnatal period. The pattern of organism in neonatal sepsis among LBW preterm infants is changing constantly [9]. Over the period of time there is a change in the group of organisms. Among the developed countries the most common cause of neonatal sepsis are Gram-negative pathogens [10]. 
Unless proper treatment is not given neonatal septicemia can be life threatening. Neonatal sepsis present with non-specific signs and symptoms which complicates the clinical diagnosis. With availability of varies diagnostic modalities for neonatal sepsis such as total blood count, erythrocyte sedimentation rate (ESR), C-reactive protein, platelet count but gold standard is blood culture [11].

An insight about common organism and their susceptibility pattern in neonatal sepsis is required to select proper antibiotic treatment to reduce the infant mortality and morbidity. The resistance pattern vary according to geographical location, pathogen and antibiotics commonly used in the neonatal wards [12]. It has been a great challenge in the management of neonatal septicemia along with emerging drug resistance to combat infection. Ongoing review on the microbiological pattern and antibiotic resistance pattern among neonatal septicemia is highly needed [13].

The frequent emergence of drug resistance in bacteria compounds the problem among LBW preterm infants. The resistance pattern and the etiological agent of the LBW infants varies based on the geographical area. This study was undertaken to know the changing pattern of Gramnegative infection among LBW infants and to highlight on the need for surveillance on neonatal sepsis and their resistance pattern of the pathogens isolated.

\section{Materials and methods}

A total of 226 LBW infants of 425-1,600 g born at the Chinese hospital between Jan 2017-Oct 2019 and those infants who survived more than $14 \mathrm{~h}$ were included in our study. 100 control group includes normal birth weight infants of 2,400-3,500 g with sepsis were included.

Blood or cerebrospinal fluid (CSF) cultures were obtained from LBW infants after development of symptoms. 2-3 blood cultures were taken from infants at late stage diagnosed with sepsis. All samples were collected before administration of antibiotics. All the demographic details such as age, sex, previous pregnancy were taken from the hospital record. All the parents were informed about the study and informed consent were obtained from the parents. Only those who gave consent were included in our study. Babies on prior antibiotic treatment and with congenital anomalies were excluded from the study. The study was approved by Institutional Ethical Board (IRB29/10456/2017).

The samples were processed according to standard protocol. The criteria included for evaluation are sepsis criteria, age of gestation, age at onset of symptoms, sex, birth weight, organism isolated and the pattern of antibiotic susceptibility. Those LBW infants with fever, apnoea, trachypnea, lethargy, poor feeding were diagnosed as to have sepsis. Other biochemical investigations were done like total white blood cell count, differential blood count, total neutrophil ratio, ESR, C-reactive protein, CSF examination, blood glucose level estimation, serum calcium, creatinine, electrolyte were tested.

A total of $2 \mathrm{~mL}$ of blood were inoculated into Bactec pediatric blood culture bottles and loaded into BACT/ALERT 460 blood culture system the samples were incubated for seven days. Positive bottles were sub-cultured on to trypticase soy agar with 5\% sheep blood, eosin methylene blue (EMB) agar, chocolate agar, Mac Conkey agar (HiMedia), daily for seven days. Blood agar and chocolate agar plates were incubated in $5-10 \% \mathrm{CO}_{2}$ using Candle jar at $37^{\circ} \mathrm{C}$ for $24-48 \mathrm{~h}$. Mac Conkey plates were incubated aerobically. After seven days of incubation blood culture bottles showing no growth were reported as negative. The bacterial species were identified by conventional methods. All the blood samples collected were processed following standard guidelines [14].

The antimicrobial testing was performed following standard guidelines by Kirby Bauer disc diffusion method. The following antibiotics were used ampicillin/sulbactam $(10 / 10 \mu \mathrm{g})$, gentamicin $(10 \mu \mathrm{g})$, cefotaxime $(30 \mu \mathrm{g})$, ceftriaxone $(30 \mu \mathrm{g})$, ofloxacin $(5 \mu \mathrm{g})$, meropenem $(10 \mu \mathrm{g})$, amikacin $(30 \mu \mathrm{g})$, cotrimoxazole $(25 \mu \mathrm{g})$, piperacillin/tazobactam $(100 / 10 \mu \mathrm{g})$. Escherichia coli ATCC25922 and Staphylococcus aureus ATCC 25923 were used as quality control standards for antimicrobial susceptibility testing [15].

\section{Statistical analysis}

The data collected were analyzed by SPSS software version 20.0 to determine the changes in overall sepsis rate, rate of infection specific to pathogen were analyzed.

\section{Results}

Out of 226 blood cultures received from LBW infants 106 (46.9\%) showed blood culture positive, 31 (13.7\%) grew contaminants and $89(39.38 \%)$ showed no growth for bacteria. Out of 100 blood cultures from control group 46 (46\%) showed positive for blood culture. A total of $28(28 \%)$ grew contaminant, 26 (26\%) showed no growth (Figures 1, 2). The mean gestational age for infants with neonatal septicemia was 25.4 weeks (95\% C.I: $24.4,25.8)$ and 27.1 weeks (95\% C.I: 27.5, 28.4), respectively. Sepsis caused by Gram-negative microorganisms higher than caused by Gram-positive microorganisms ( $\mathrm{p}<0.0001)$.

\section{Blood culture postive among study group population}

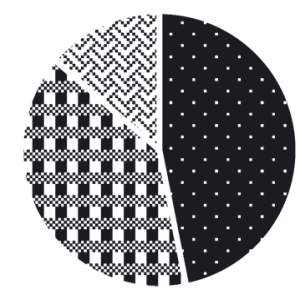

- Blood culture positive $\quad$ No growth $\approx$ contaminant

Figure 1: Distribution of blood culture positive among study group population. 


\section{Distribution of blood culture positive among control group population}

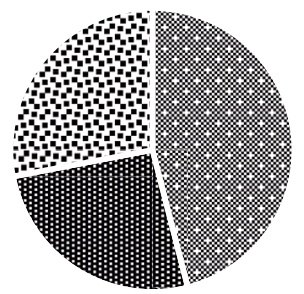

* Blood culture positive $\quad$ No growth $\quad$ contaminant

Figure 2: Distribution of blood culture positive among control group population.

Out of 106 blood culture positive neonates 66 (62.26\%) were inborn, 40 (37.74\%) were out born neonates (Table 1). Of which 57 (53.77\%) were males and 49 (46.23\%) were females (Table 2). Among control group out of 46 positive blood culture 27 (58.7\%) were inborn and 19 (41.30\%) were out born neonates (Table 1). Among 46 neonates 28 $(60.86 \%)$ were male and $18(39.14 \%)$ were females (Table 2).

Neonatal septicemia caused by Gram-negative isolates were 98 (92.45\%) compared to Gram-positive organism 8 $(7.55 \%)$ causing neonatal septicemia (Figure 3). Sepsis caused by Gram-negative microorganisms higher than caused by Gram-positive microorganisms ( $\mathrm{p}<0.0001)$. Out of 106 blood culture seven bacterial isolates were isolated. E. coli 52 (49\%), Klebsiella pneumoniae 28 (26.4\%), Enterobacter spp. 12 (11.32\%), Pseudomonas aeruginosa 4 (3.7\%), Acinetobacter spp. 2 (1.88\%), methicillin-resistant S. aureus (MRSA) 5 (4.7\%) and Staphylococcus epidermidis $3(2.8 \%)$ were isolated from our study (Table 3$)$.

Table 1: Birth parameters between study and control group population.

\begin{tabular}{lrr}
\hline Parameters & $\begin{array}{r}\text { Study group } \\
(\mathrm{n}=106)\end{array}$ & $\begin{array}{r}\text { Control group } \\
(\mathrm{n}=46)\end{array}$ \\
\hline Inborn neonates & $66(62.26 \%)$ & $27(58.7 \%)$ \\
Out-born neonates & $40(37.74 \%)$ & $19(41.30 \%)$ \\
\hline
\end{tabular}

Table 2: Sex wise distribution among culture positive in study and control group population.

\begin{tabular}{lrr}
\hline Sex & $\begin{array}{r}\text { Study population } \\
(\mathbf{n = 1 0 6 )})\end{array}$ & $\begin{array}{r}\text { Control population } \\
(\mathbf{n}=\mathbf{4 6})\end{array}$ \\
\hline Male & $57(53.77 \%)$ & $28(60.86 \%)$ \\
Female & $49(46.23 \%)$ & $18(39.14 \%)$ \\
\hline
\end{tabular}

Distribution of isolates in Neonatal septicemia in our study

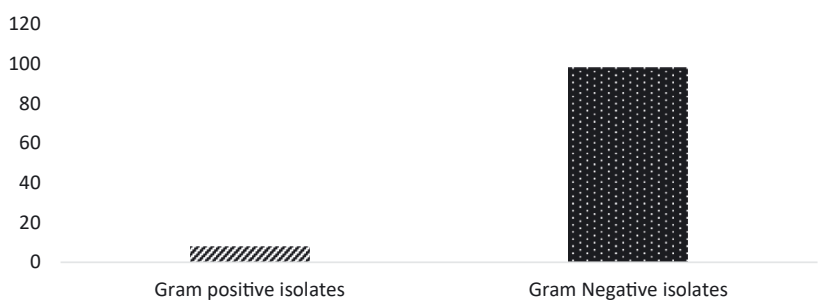

Figure 3: Distribution of isolates in neonatal septicemia in our study.

Table 3: List of Gram-negative organism isolated from study group organism.

\begin{tabular}{lr}
\hline Organism & Number isolated \\
\hline E. coli & $52(49 \%)$ \\
K. pneumoniae & $28(26.4 \%)$ \\
Enterobacter spp. & $12(11.32 \%)$ \\
$P$. aeruginosa & $4(3.7 \%)$ \\
Acinetobacter spp. & $2(1.88 \%)$ \\
MRSA & $5(4.7 \%)$ \\
S. epidermidis & $3(2.8 \%)$ \\
\hline
\end{tabular}

MRSA, methicillin-resistant Staphylococcus aureus.

Among control group neonatal septicemia Gramnegative isolates were $39(84.8 \%)$ isolated and Grampositive isolates were $5(10.9 \%)$ and candida $2(4.3 \%)$ were isolated. Among the control group 18 (39\%) were E. coli, 12 (26.08\%) was Enterobacter, 8 (17.39\%) was K. pneumoniae, $1(2.17 \%)$ was Pseudomonas, $2(4.34 \%)$ were MRSA, 3 (6.52\%) were $S$. epidermidis and $2(4.34 \%)$ were Candida spp. In our study E. coli (46.05\%) was isolated being the most common pathogen from study and control group population (Table 4).

The following antibiotics were used ampicillin/sulbactam $(10 / 10 \mu \mathrm{g})$, gentamicin $(10 \mu \mathrm{g})$, cefotaxime $(30 \mu \mathrm{g})$, ceftriaxone $(30 \mu \mathrm{g})$, ofloxacin $(5 \mu \mathrm{g})$, meropenem $(10 \mu \mathrm{g})$,

Table 4: List of Gram-negative organism isolated from control group organism.

\begin{tabular}{lr}
\hline Organism & Number isolated \\
\hline E. coli & $18(39 \%)$ \\
K. pneumoniae & $8(17.39 \%)$ \\
E. spp. & $12(26.08 \%)$ \\
P. aeruginosa & $1(2.17 \%)$ \\
MRSA & $2(4.34 \%)$ \\
S. epidermidis & $3(6.52 \%)$ \\
Candida & $2(4.34 \%)$ \\
\hline
\end{tabular}

MRSA, methicillin-resistant Staphylococcus aureus. 
amikacin (30 $\mu \mathrm{g})$, cotrimoxazole $(25 \mu \mathrm{g})$, piperacillin/ tazobactam $(100 / 10 \mu \mathrm{g})$. E. coli ATCC25922 and S. aureus ATCC 25923 were used. In our study highest resistance was observed for cephalosporin group of antibiotics with 58\% resistance followed by penicillin group with 52\% resistance. Aminoglycoside group showed least resistance with $22 \% \beta$-lactam antibiotics showed highest sensitivity of $96 \%$ sensitivity (Table 5).

In control group penicillin group of antibiotics showed highest resistance with $62 \%$ followed by cephalosporin group with $54 \%$ resistance. $18 \%$ resistance was observed for aminoglycoside group. Beta lactam group of antibiotics showed highest sensitivity of $97.5 \%$ (Table 6).

\section{Discussion}

In the neonatal intensive care unti (NICU) the leading cause of infant mortality and morbidity is neonatal septicemia, especially LBW infants has higher mortality and morbidity rate $[16,17]$. Overall infection rate has changed during past 13 years with decline in Gram-positive infection and raise in Gram-negative bacterial infection since 2000. The most troubling and important observation in our study is Gramnegative infections are high among LBW infants with septicemia. The most frequently isolated pathogen in our study is $E$. coli which is similar to the study by Stoll et al. 2005 [8]. The incidence of infection in NICU varies from 6 to $25 \%$ in USA, recent studies have reported $48 \%$ of neonatal sepsis [18]. Haque et al. [19] also reported a higher incidence of neonatal sepsis up to $48 \%$. In our study we have an overall incidence of $46.9 \%$ correlating with other

Table 5: Resistance profile of Gram-negative organism among study group population.

\begin{tabular}{lr}
\hline List of antibiotics class & Resistance, $\%$ \\
\hline Cephalosporin & $58 \%$ \\
Penicillin & $52 \%$ \\
Aminoglycoside & $22 \%$ \\
$\beta$-Lactam antibiotics & $4 \%$ \\
\hline
\end{tabular}

Table 6: Resistance profile of Gram negative isolates among control group population.

\begin{tabular}{lr}
\hline List of antibiotics class & Resistance, $\%$ \\
\hline Penicillin & $62 \%$ \\
Cephalosporin & $54 \%$ \\
Aminoglycoside & $18 \%$ \\
$\beta$-Lactam antibiotics & $3 \%$ \\
\hline
\end{tabular}

studies. Al Shamahy et al. 2012 [20] reported 57\%. The variation in the positivity of blood culture among neonatal septicemia may be due to difference in sample size, prior antibiotic administration before sample collection, infection due to anaerobic organisms are the reasons [21].

In our study neonatal septicemia was more common among males with 53.77 and $46.23 \%$ females in control group population also showed male predominance with $60.86 \%$ male and $39.14 \%$ female which coincides with other studies showing septicemia incidence rate with male ranging from 59-82\% [22]. A further investigation on the pathophysiology of sepsis in neonates is required to get an insight in the diagnosis, treatment and management of the sepsis [13].

In our study majority of the isolates were Gramnegative with $92.45 \%$ which is high compared to Thapa and Sapkota et al. 2019 [13] had reported $69.6 \%$ of Gramnegative isolates from their study other studies had higher incidence of Gram-positive pathogens being predominant in their study [23]. Neonatal septicemia causative agents will change from time to time and vary according to geographical locations [24].

In our study the commonest and predominant pathogen is $E$. coli which is similar to study by Kumaravel et al. 2016 [25]. In a study conducted by Mutlu et al. 2011 also reported $E$. coli to be the most common organism isolated among neonatal sepsis. A high degree of antimicrobial resistance is observed among Gram-negative microorganism.

Couto et al. 2007 [26] had reported highest resistance to third generation cephalosporin for E. coli and Klebsiella spp. ranging from 19 to $64 \%$, which is high compared to our study with 58\% resistance observed for cephalosporin group of antibiotics. Control group also showed 54\% resistance for cephalosporin group.

In our study all the Gram-negative isolates showed highest resistance for cefotaxime antibiotic among the cephalosporin group. Penicillin group also showed highest resistance with $52 \%$ in study group and $62 \%$ resistance in control group population which is similar to Aurangzep and Hamed et al. studies [27]. Least resistance was observed for aminoglycoside group of antibiotics with resistance of $22 \%$ in study group and $18 \%$ in control group which coincides with the study by Mutlu et al. 2011 [28]. The empirical antibiotic administration as treatment is not effective against all microorganisms. Culture reports with antibiotic susceptibility should be obtained as early as possible.

Higher resistance reported in last five years may be due to emerging resistant bacterial strain because of inappropriate and irrational use of antibiotics in many private clinics and primary health care facilities [29]. Firm hospital infection control policy and hand hygiene prevent transmission of infection especially in neonatal ward. Expanded 
surveillance of the microorganism involved in LBW infants and data including infants of all birth weights is required to get clear data on the infections [13].

\section{Limitations}

More clinical studies and epidemiological data are required to curb the changes in neonatal sepsis among LBW infants.

\section{Conclusions}

High degree and emerging antibiotic resistance are significantly associated with mortality and morbidity in neonatal sepsis infants. Routine antimicrobial surveillance and periodic antibiotic cycling with national antibiotic policy helps to decrease the burden of antibiotic resistance in LBW infants with neonatal sepsis.

Research funding: None declared.

Author contributions: All authors have accepted responsibility for the entire content of this manuscript and approved its submission.

Competing interests: Authors state no conflict of interest. Informed consent: Informed consent was obtained from all individuals included in this study.

Ethical approval: The study was approved by Institutional Ethical Board (IRB29/10456/2017).

\section{References}

1. Stoll BJ, Hansen N, Fanaroff AA. Changes in pathogens causing early-onset sepsis in very-low-birth-weight infants. N Engl J Med 2002;347:240-7.

2. Klein JO. Bacterial sepsis and meningitis. In: Remington JS, Klein JO, editors. Infectious diseases of the fetus and newborn infant, 5th ed. Philadelphia, PA: W. B. Saunders Co.; 2000:943-98 pp.

3. Cordero L, Sananes M, Ayers LW. Bloodstream infections in a neonatal intensive-care unit: 12 years' experience with an antibacterial control program. Infect Control Hosp Epidemiol 1999;20:242-6.

4. Stoll BJ, Hansen N, Fanaroff AA, Wright LL, Carlo WA, Ehrenkranz RA. Late-onset sepsis in very low birth weight neonates: the experience of the NICHD Neonatal Research Network. Pediatrics 2002;110:285-91.

5. Stoll BJ, Gordon T, Korones SB, Shankaran S, Tyson JE, Bauer CR. Late-onset sepsis in very low birth weight neonates: a report from the National Institute of Child Health and Human Development Neonatal Research Network. J Pediatr 1996;129:63-71.
6. Haque KM. Neonatal sepsis in very low birth weight preterm infants: part 1: review of patho-physiology. J Med Sci 2010;3: 1-10.

7. Stoll BJ, Hansen N, Fanaroff AA, Wright LL, Carlo WA. Late-onset sepsis in very low birth weight neonates: the experience of the NICHD Neonatal Research Network. Pediatrics 2002;110: 285-91.

8. Stoll BJ, Hansen NI, Higgins RD, Fanaroff AA, Duara S. Very low birth weight preterm infants with early onset neonatal sepsis: the predominance of Gram-negative infections continues in the National Institute of Child Health and Human Development Neonatal Research Network, 2002-2003. Pediatr Infect Dis J 2005;24:635-9.

9. Prabhu K, Bhat S, Rao S. Bacteriologic profile and antibiogram of blood culture isolates in a pediatric care unit. J Lab Phys 2010;2:85.

10. Zea-Veraand A, Ochoa TJ. Challenges in the diagnosis and management of neonatal sepsis. J Trop Pediatr 2015;61:1-13.

11. Rahman S, Hameed A, Roghani MT. Multidrug resistant neonatal sepsis in Peshawar, Pakistan. Arch Dis Child 2002;87:52-4.

12. Premlatha DE. The bacteriological profile and antibiogram of neonatal septicemia in a tertiary care hospital. Int J Sci Technol Soc 2014;10:451-5.

13. Thapa S, Sapkota LH. Changing trend of neonatal septicemia and antibiotic susceptibility pattern of isolates in Nepal. Int J Pediatr 2019;1:1-7.

14. Winn WC, Allen SD, Janda WN. Konemans color atlas and textbook of diagnostic microbiology, 6th ed. Philadelphia, PA, USA: Lippincott; 2006.

15. Collee JG, Hayward NJ, Marr W. Blood culture. In: Cruickshank K, Duguid JP, Marmion BP, Swain RHA, editors. Medical microbiology, 12th ed. Edinburgh, Scotland, UK: Livingstone; 1975, 2:162-4 p.

16. Stoll BJ, Hansen N, Fanaroff AA, Wright LL, Carlo WA, Ehrenkranz RA. Late-onset sepsis in very low birth weight neonates: the experience of the NICHD Neonatal Research Network. Pediatrics 2002;110:285-91.

17. Benjamin DK, DeLong E, Cotten CM, Garges HP, Steinbach WJ, Clark RH. Mortality following blood culture in premature infants: increased with Gram-negative bacteremia and candidemia, but not Gram-positive bacteremia. J Perinatol 2004;24:175-80.

18. Sobaih BH, Al-Mandeel H. Early and late onset neonatal sepsis in very low birth weight infants in a Tertiary Center in Saudi Arabia. J Neonatal Biol 2014;3:159.

19. Haque KN, Chagia AH, Shaheed MM. Half a decade of neonatal sepsis, Riyadh, Saudi Arabia. J Trop Pediatr 1990;36:20-3.

20. Al-Shamahy HA, Sabrah AA, Al-Robasi AB, Naser SM. Types of bacteria associated with neonatal sepsis in Al Thawra University Hospital, Sana'a, Yemen, and their antimicrobial profile. Sultan Qaboos Univ Med Sci J 2012;12:48-54.

21. Raha BK, Baki MA, Begum T, Nahar N, Jahan N, Begum M. Bacteriological profile \& outcome of neonatal sepsis in a Tertiary Care Hospital. Med Today 2014;26:18-21.

22. Schreiber JR, Berger M. Intravenous immune globulin therapy for sepsis inpremature neonates. J Pediatr 1992;121:401-4.

23. Nayak S, Kumar V, Pai A, Ganesh H, Rai R, Sanjeev H. Distribution of microorganisms in neonatal sepsis and antimicrobial 
susceptibility patterns in a tertiary care hospital. Arch Med Health Sci 2014;2:136-9.

24. Mishra A, Mishra S, Jaganath G, Mittal RK, Gupta PK, Patra DP. Acinetobacter sepsis in newborns. Indian Pediatr 1998;35:27-32.

25. Kumaravel KS, Rameshbabu B. A study of the bacteriological profile and antibiotic sensitivity in neonatal septicaemia. Int J Contemp Med Res 2016;3:1830-1.

26. Couto RC, Carvalho EA, Pedrosa TM, Pedroso ER, Neto MC, Biscione FM. A 10-year prospective surveillance of nosocomial infections in neonatal intensive care units. Am J Infect Contr 2007; 35:183-9.
27. Aurangzeb B, Hameed A. Neonatal sepsis in hospital-born babies: bacterial isolates and antibiotic susceptibility patterns. J Coll Phys Surg Pak 2003;13:629-32.

28. Mutlu M, Aslan Y, Saygin B, Yilmaz G, Bayramoğlu G, Köksal I. Neonatal sepsis caused by Gram-negative bacteria in a neonatal intensive care unit: a six years analysis. Hong Kong J Paediatr 2011;16:253-7.

29. Rao P, Sowmya KN, Shrikala B, Radhakrishna M, Keerthiraj B. A spectrum of bacterial pathogens and its antibiotic susceptibility pattern isolated from neonatal sepsis in an NICU in a Government Pediatric Hospital. Int Res J Biol Sci 2015;4:50-4. 